\title{
Parametric aspects of tactile resolution *
}

\author{
AMIRAM CARMON + and ARTHUR L. BENTON \\ Neurosensory Center and Departments of Neurology and Psychology \\ University of Iowa, Iowa City, Iowa 52240
}

Three parameters of tactile resolution (area, force, and pressure of stimulation) were investigated in three experiments. A two-point discrimination task was presented by means of an electromechanical stimulator permitting precise control of all relevant stimulus variables. The results indicated a positive linear effect of area, a curvilinear effect of force, and no effect of pressure on the threshold for resolution of two tactile stimuli. The findings are in agreement with data on the influence of the same parameters in visual resolution.

Since the pioneering studies on the two-point threshold by Weber (1851) and Czermak (1879), little effort has been devoted to investigating parametric aspects of spatiotactile rf solution. Instead, the two-point task was used mainly to test attention, ctrebral status, etc. The effect of stimulus intensity on the two-point threshold was investigated by Cook and von Frey (1911), who reported that increase of the stimulus intensity leads to a lower two-point threshold. Support for this finding came from von Békésy (1959), though he did not present detailed data in his report. Malmud and Nygard (1931), on the other hand, concluded that opposite relations existed between spatiotactile resolution and stimulus intensity, i.e., that weak stimuli were separated by the skin senses better than were strong ones.

We have not been able to find any references in the literature to studies of the effects on tactile resolution of systematic variations of other variables such as the area of stimulation and the pressure of the stimulus (force per area). This lack was encountered during the course of other studies in this laboratory (Carmon, 1968), which utilized the tactile resolution function in investigating the spatial interaction of tactile inhibition and excitation. Hence, in order to establish some necessary parametric data with regard to spatial resolution in the somatosensory system, a series of studies on the influence of some stimulus variables on the two-point threshold was undertaken, the results of which are reported here. The data were obtained with the aid of a controlled mode of stimulation, changing only one stimulus variable at a time and keeping constant the temporal duration and simultaneity of

\footnotetext{
*This investigation was supported by Research Grant NB-00616 and Program-Project Grant NB-03354 from the National Institute of Neurological Diseases and Blindness. Neurosensory Center Publication No. 138.

+Present address: Department of Neurology, Hebrew University, Hadassah Medical School, Jerusalem. Israel.
}

different pairs. The rods were supported in nylon sleeves, with their lower parts projecting through a hole and permitting nearly frictionless movements up to a maximum of $0.3 \mathrm{in}$. The sleeves were attached to a scissor-like device, and the distance between the two rods was controlled by using a calibrated scale on the opposite side of the scissors, which could be varied from 0.1 to 1.5 in. The center pivot of the scissors was attached to the center of a plunger of a solenoid with a lifting capacity of $5 \mathrm{lbs}$. The power supply to the solenoid was controlled by a 124 stimulation throughout all the experiments.

\section{EXPERIMENT 1:}

\section{INFLUENCE OF AREAL SIZE OF STIMULATION ON TACTILE RESOLUTION}

\section{Method}

Subjects. Five university students participated in this experiment. All had intact somatosensory mechanisms, as judged by routine neurological examination. Each $\mathbf{S}$ had had 10 previous experimental sessions with the task of two-point discrimination.

Apparatus. An electromechanical tactile stimulator (Carmon \& Dyson, 1967) that provided for control of pressure, duration, and locus of application of two simultaneously presented tactile stimuli was utilized. The stimuli were the flat circular surfaces of a pair of aluminum rods weighing $8 \mathrm{~g}$ each. The rods varied in length from 2.5 to 4 in.; their upper part had a diameter of 0.25 in., and the diameter of the eccentric lower $1 \mathrm{in}$. of the length was varied for the
Hunter time-interval cycler. S's hand rested on a vertically adjusted base under the solenoid, which was mounted on horizontally moving slides. A curtain in front of the apparatus obstructed the S's view of his hand.

Five pairs of rods, with stimulating areas of $0.003,0.007,0.028$, and $0.049 \mathrm{sq}$ in., respectively, were used.

Procedure. Five experimental sessions were conducted with each $\mathbf{S}$. The method of constant stimuli was used to determine the number of two-point resolutions. Forty stimulations for each pair of stimuli, in distances varying in a random order from 0.1 to $0.8 \mathrm{in}$. (in 0.1 -in. steps), were given in each session, 20 for each hand. The order of presentation of the stimuli was counterbalanced.

In each session, the $S$ sat facing the $E$ and inserted his hand, with the palm up, through the curtain and rested it on the vertically moving base. The $\mathrm{E}$ moved the base upward until the desired area for stimulation on the palm was 0.1 in. beneath the
(Woight of stimulus : $8 \mathrm{gms}$ )

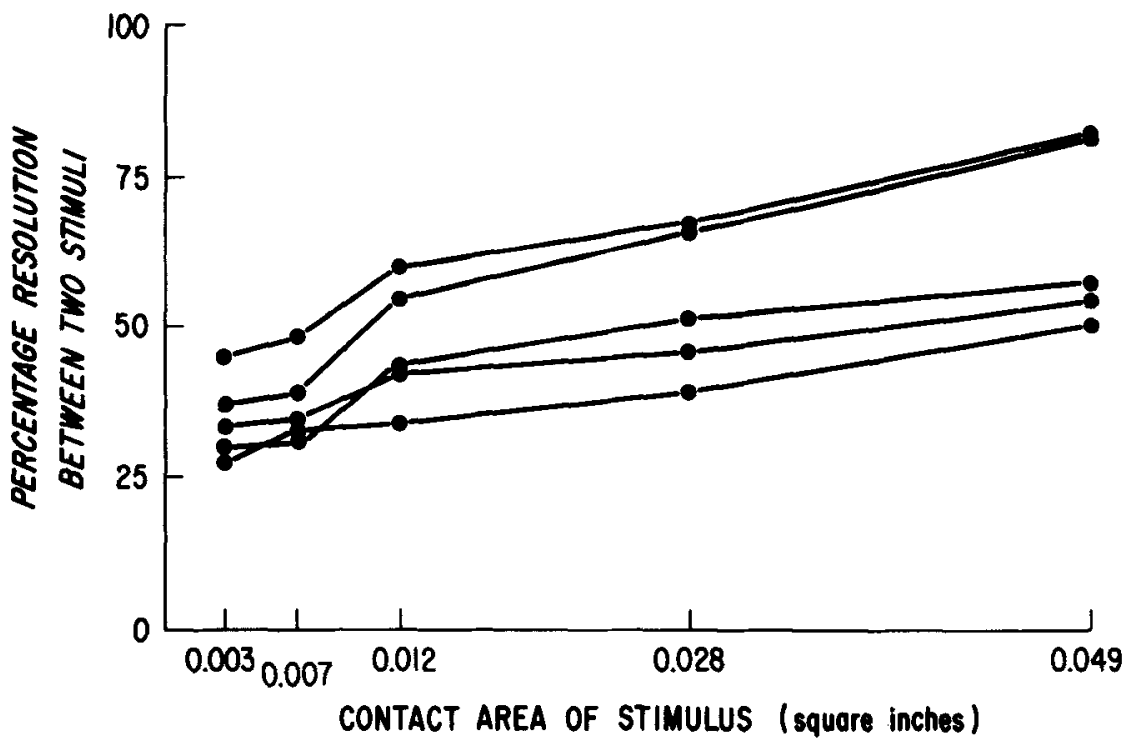

Fig. 1. Tactile resolution as a function of stimulus area; individual data for five Ss. 


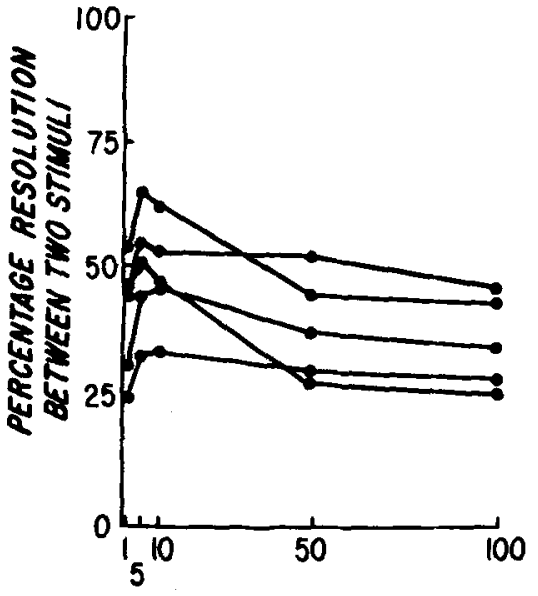

WEIGHT OF STIMULI (OrOMB)

Fig. 2. Tactile resolution as a function of stimulus force (weight); individual data for Group 1 (see text).

stimulating tips. Horizontal adjustments were made by manipulation of the horizontally moving supporting slides and by rotatory movement of the central pivot of the scissors. By activating the interval timer, the current to the solenoid was interrupted and the stimulating rods descended for $0.3 \mathrm{in}$. They became supported by the palm, their weights resting on the area selected for stimulation until they were lifted again by resumption of the current.

The area chosen for stimulation was in the center of the palm; it was kept constant by the use of printed grids. The stimuli were applied in a transverse plane. The interval timer was programmed for stimulations of $1 \mathrm{sec}$, with an interstimulus interval of $5 \mathrm{sec}$ in each set of 20 stimuli. The actual duration of the stimulation was $1.05 \mathrm{sec}$ because of a short lag in the lift of the solenoid. In each session (approximately $1 \mathrm{~h}$ ) 200 stimulations were applied. Thus, each $S$ received a total of 1,000 stimulations during the experiment.

The Ss were required to make a forced choice between a response of "one" and "two" for each stimulation. The instructions were to give a response of "two" only when two distinctly separate touches were felt. Single stimuli were not presented for two reasons: (1) In previous experiments with stimulators of the same size and weight, it had been shown that these Ss were not able to differentiate in any way between a single presentation and a double stimulation which was felt as single; and $(2)$ the interest here was in a comparison between the number of resolutions achieved with the different stimuli and not in the absolute level of performance.
Results

The data for the individual $S$ s are presented graphically in Fig. 1. Examination of the figure shows that resulted in a nearly linear increase in the percentage of resolutions. Increase of the area from 0.003 to $0.007 \mathrm{sq}$ in., which was the smallest absolute increase, did not increase the number of resolutions as much as did larger absolute increases. The effect of area was tested by a repeated measures analysis of variance and was found to be significant $(F=37.71, d f=4,16$, $\mathrm{p}<.01$ ).

A greater number of resolutions was made with successively larger areas at almost every point along the distance scale. This pattern was found in each

\section{EXPERIMENT 2: \\ INFLUENCE OF FORCE (WEIGHT) OF STMMULATION ON TACTILE RESOLUTION}

Method

The apparatus and the method were identical to that of the first experiment. The five $S s$ from the former experiment (Group 1) and five additional Ss, who were given two training sessions each (Group 2), served in the experiment. The stimuli used in this experiment were aluminum rods with flat surfaces and increase in the areal size of the stimuli of the Ss. with weights of $1,5,10,50$, and $100 \mathrm{~g}$, respectively. Group 1 received a total of 1,000 stimulations with rods with stimulating area of $0.012 \mathrm{sq}$ in. For Group 2, two area sizes for stimulation were used: 0.003 and $0.049 \mathrm{sq}$ in. Each $S$ in this group received a total of 800 stimulations, 400 for each area size and 80 for each weight.

\section{Results}

The data are plotted in Figs. 2 and 3. The data for Group 1 (Fig. 2) show that, while there was an initial increase in the percentage of resolution with an increase in force (weight) of the stimuli, further increase in force resulted in a decrease in the percentage of resolutions. The two curves in Fig. 3 for each $S$ of Group 2 show their performances for the two areas. Inspection of the curves shows that increase in the force of the stimulus resulted initially in an increase in the percentage of resolutions in all Ss. With the smaller areas, there was a decrease in the percentage of resolutions following further increase of the force. With the larger areas, however, such a decrease was not observed in two Ss. The force with is achieved was consistently greater in the case of the larger than in the case of the smaller area. Since, in both which maximal number of resolutions

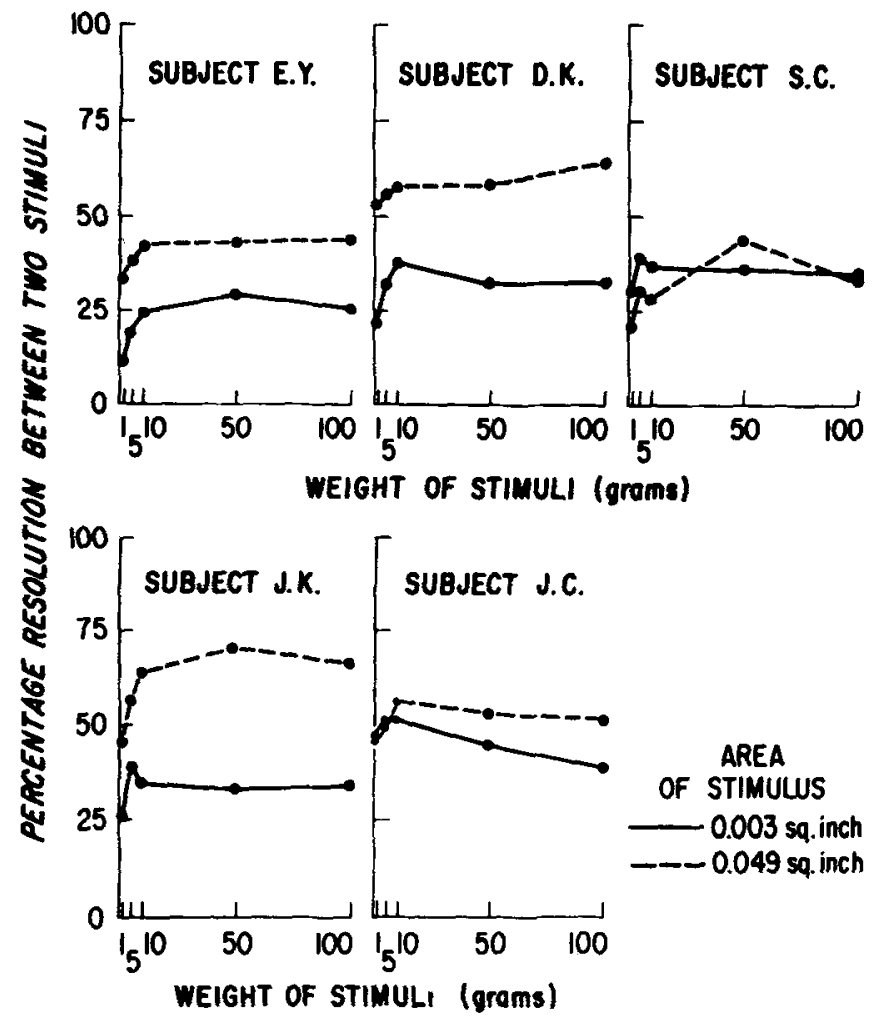

Fig. 3. Tactile resolution as a function of stimulus force (weight) and stimulus area; individual data for Group 2 (see text). 


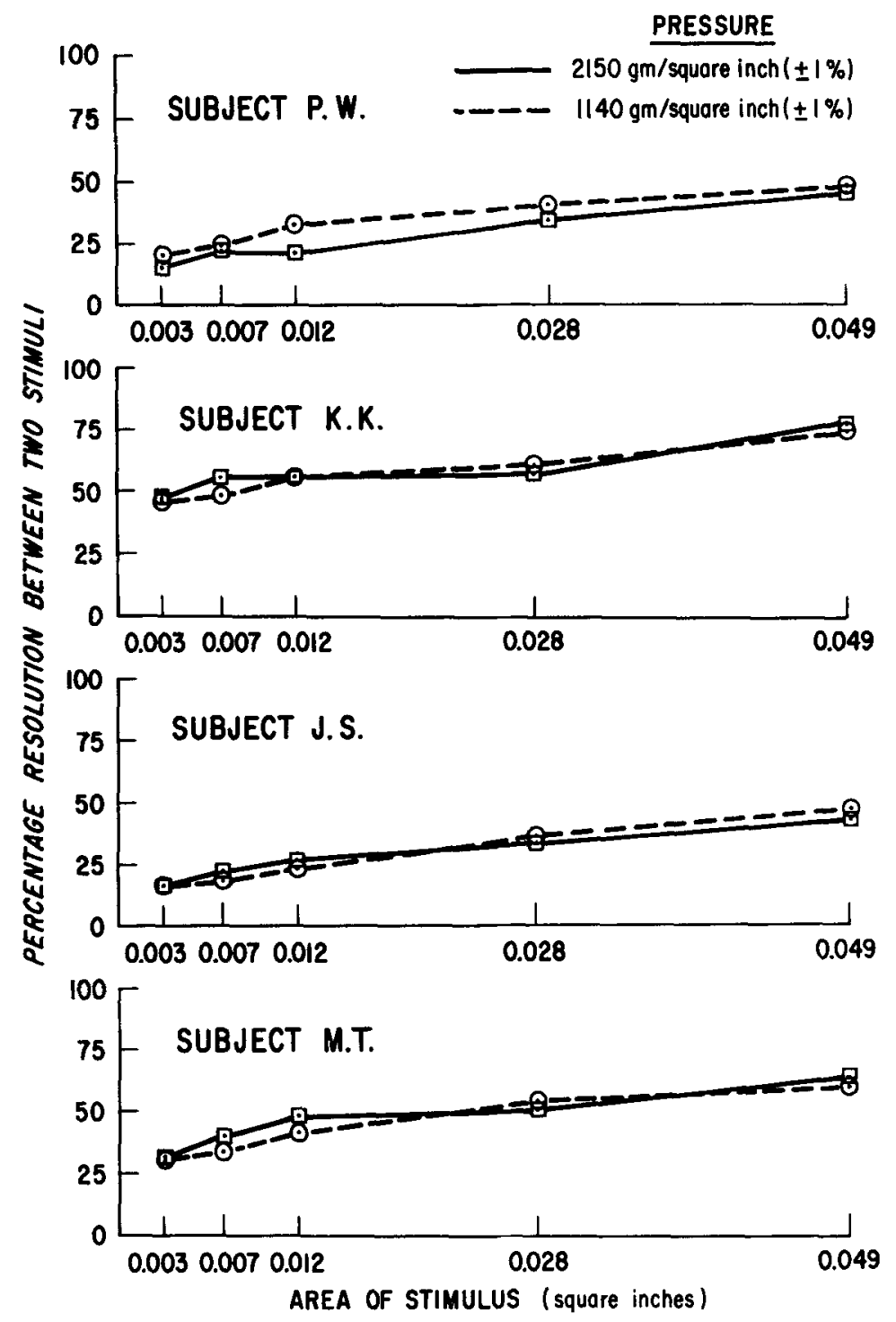

Fig. 4. Tactile resolution as a function of pressure and stimulus area; individual data for four Ss.

groups, the curvilinear relationship between the stimulus force and the number of resolutions were similar only in form but not in the location on the force continuum, no statistical analysis of the results was carried out.

EXPERIMENT 3:

EFFECT OF PRESSURE AND

AREAL SIZE OF STIMULATION Method

ON TACTILE RESOLUTION

Four the five Ss who participated in the first experiment served in this experiment. The apparatus and the method were identical to those of the previous two experiments. The same stimuli used in the first experiment were also used here, with the exception that their weights were varied so that two levels of pressure (force per area) were used-1,140 and $2,150 \mathrm{~g} / \mathrm{sq}$ in. The use of this range of
The effect of the change of the area was significant $(F=48.04 ; \mathrm{df}=4,27$; $\mathrm{p}<.01$ ).

\section{DISCUSSION}

The findings of the first experiment indicate that there is a positive linear relationship between increase of the area of stimulation and increase in the resolving power of the somatosensory system. The findings can be explained in terms of the mutal interaction of inhibition and excitation within a stimulated area. Studies on interaction between single receptors in the eye showed that there exists mutual inhibition between neighboring receptors. The inhibition is inversely related to the distance between the receptors, while also being directly related to the intensity of excitation of the inhibiting receptor (Ratliff \& Hartline, 1959). The model of lateral inhibition was used by one of us (Carmon, 1968) to investigate the effect of stimulus configuration on spatiotactile resolution. According to the model, dispersion of a constant amount of intensity over more receptors will result in a decrease of the mutual inhibition within the stimulated area, and in an increase of the stimulation gradient between the stimulated areas and the gap between them. Thus, there will be better resolution between two spatially placed stimuli when the area of the stimuli is increased.

The same interpretation can be applied to the results of the second experiment in which the stimulus intensity was varied. The decrease in resolution with small areas and high stimulus intensity might be explained in terms of the inhibition exerted by one stimulated area on another which leads to a decrease in the gradient between the stimulated and nonstimulated areas. When the areal size of stimulation was increased, as in the second part of Experiment 2, the stimulus intensity per area was decreased and thus the inhibitory effect which transcends the interstimulus space was lessened.

The effect of change in the area of stimulation on tactile resolution was found to be stronger than the effect of change in intensity. As shown in Experiment 3, the first effect may cancel the second. This finding suggests that the number of receptors stimulated is more critical for resolution than the amount of suprathreshold intensity with which they are stimulated. (The absolute threshold in our Ss was much lower than any intensity used in the present experiments.)

The lack of significant interaction between pressure and area observed in Experiment 3 was probably due to the fact that the stimulus intensities used 
in order to achieve pressure differences did not differ enough in any given stimulus area to produce consistent changes in resolution thresholds. This is true at least in the case of the stimuli with an area of $0.049 \mathrm{sq}$ in., which had intensities of 53.85 and $105.35 \mathrm{~g}$, respectively. Such a difference failed to produce consistent differences in the resolution level in Experiment 2.

A recent study by Vierck and Jones (1968) showed that in a tactile size discrimination task the pressure of application of cylindrical stimuli did not affect the results. When performance was measured in terms of detection of linear differences in size, it was found to be superior to the detection of an existing gap between or within tactile stimuli. These findings support the notion that the number of receptors stimulated and not the intensity of stimulation is the critical factor in spatiotactile resolution.

Indirect support for the present findings, though from a different sensory modality, comes from the parametric study of Fry and Cobb (1939) on the effect of area and intensity of discriminanda on visual resolution. In this study, the experimental task was the resolution between two parallel bars in a dark field. Increase in the width of the bars improved the visual resolution significantly. Increase of the intensity of narrow bars (168 min) had an initial improving effect but, when it exceeded a certain optimal value, the effect was just the opposite, i.e., decrease of the ability of the $S$ to resolve between the bars. However, increase of the intensity of wide parallel bars $(1,000 \mathrm{~min})$ resulted in improvement in the resolution which then rapidly approached a plateau. These results are similar to the psychophysical functions found in the present study.

The correlation between the size of the area of stimulation and spatiotactile resolution merits a final comment. Weber (1851) and Czermak (1879) demonstrated that there are differences in the spatial resolution of tactile stimuli in various zones of the body. Czermak tried to correlate this with the "stretch" of the surface of the skin, maintaining that discrimination in a member having less skin surface (e.g., finger vs hand) is better since the receptors have a higher density per unit area. However, on the basis of studies of cortical representation, the resolution threshold can be related to the relative size of the representative cortical area for the individual zones. This suggests that resolution might depend on the number of cortical cells excited. Even if this is only a partial explanation, it may shed some light on the functions under consideration. Presumably, with a larger area, the number of cortical cells excited is larger.

\section{REFERENCES}

CARMON, A. The role of contrast in tactile resolution. Perception \& Psychophysics, 1968, 3, 241-245.

CARMON, A., \& DYSON, J. A. New instrumentation for research on tactile sensitivity and discrimination. Cortex, $1967,3,406-418$

COOK, H. D., \& von FREY, M. Der Einfluss der Reizstarke auf den Wert der simultanen Raumschwelle der Haut. Zeitschrift für Biologie, 1911, 56. 537-57.

CZERMAK, J. N. Beiträge zur Physiologie des Tastsinnes. In: Gesammelte Schriften. Vol. 2. Leipzig: Engelman, 1879. Pp. 303-351.

FRY, G. A., \& COBB, P. W, Visual discrimination of two parallel bright bars in a dark field. American Journal of Psychology, 1939, 49, 76-81.

MALAMUD, W., \& NYGARD, W. J. The role played by the cutaneous receptors in spatial perception. Journal of Nervous \& Mental Disease, 1931, 73, 465-477.

RATLIFF, F., \& HARTLINE, H. K. The responses of Limulus optic nerve fibers to patterns of illumination on the receptor mosaic. Journal of General Physiology, 1959, 42, $1241-1255$.

VIERCK, C. J., \& JONES, M. B. Size discrimination on the skin. Science, 1968 , $163,488-489$.

von BEKESY, G. Neural funneling unit along the skin and between the inner and outer hair cells of the cochlea. Joumal of the Acoustical Society of America, 1959. 31, 1236-1249.

WEBER, E. H. Die Lehre vom Tastsinne and Gemeingefühle auf Versuche gegründet. Braunschweig: Vieweg, 1851.

(Accepted for publication May 1, 1971.) 